\title{
The Diameter Distribution of the Stem Villi Arteries Does Not Discriminate Between Normal and Intra Uterine Growth Restricted Placentas
}

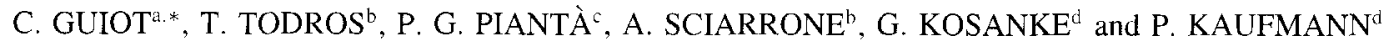 \\ ${ }^{a}$ Dip. Neuroscienze (sez. Fisiologia), Università di Torino (I); ${ }^{\circ}$ Dip. Discipline Gn. Ost, Università di Torino (1); ${ }^{\circ}$ Centro Studi \\ Dinamica dei Fluidi CNR, Torino (I); ${ }^{\mathrm{d}}$ Institut fur Anatomie der RWTH. Aachen (D)
}

(Received 13 April 1998; Revised 15 October 1998; In final form 8 December 1998)

\begin{abstract}
The distribution of the stem villi arteries and arterioles diameter was evaluated on histological sections from 6 normal (control) and 8 IntraUterine Growth Restricted (IUGR) placentas. No significant difference between the diameter distributions was found. This result can be due either to a similar pattern in vascularization or to the inadequacy of the diameter distribution in showing existing differences. Further investigation is needed to make a distinction between these two hypotheses. A multiparametric model is proposed, which predicts the diameter distribution that is expected when a few parameters (describing the vessel geometry and the tree branching structure) are varied in their normal range. The diameter distribution is shown to slightly change or not to change at all when important parameters, such as the vessel asymmetry, are changed. We conclude that the diameter distribution is insensitive to variation in certain placental structural parameters. A reliable analysis of the stem villi vasculature in normal and IUGR placentas cannot be based on the comparison of the respective diameter distributions alone.
\end{abstract}

Keywords: IUGR. villous vessels, parameter estimation, placental morphometry

\section{INTRODUCTION}

Intrauterine growth restriction (IUGR) with impeded flow in the umbilical arteries is one of the major clinical problems in pregnancy (Kingdom, Rodeck and Kaufmann, 1997). Its pathogenetic mechanisms are still poorly understood.

The prevailing views are based on the data by Trudinger et al. (1985) and Giles et al (1985) indicating a reduction in the density of small fetoplacental stem arterioles. Embolization studies conducted in sheep (Trudinger et al, 1987) supported the 'obliteration theory'.

In contrast, detailed analysis of stem villi in IUGR with absent end diastolic flow (AED) in the umbilical arteries failed to indicate any selective loss of stem vessels (Jackson et al, 1995; Macara et al, 1995). Differences could lie mainly in the

\footnotetext{
${ }^{*}$ Corresponding Author: Dip. Neuroscienze (sez. Fisiologia), Corso Raffacllo 30, I10125, Torino, Italia; Tel: +39-11-6707702;
} Fax: +39-11-6707708; E-mail: guiot@medfarm.unito.it 
contribution of the capillary bed to the vascular impedance of the fetoplacental circulation, as has been deduced from quantitative analysis in normal pregnancies (Leiser et al, 1991). This view is supported by morphometric and scanning electron microscopic studies of the terminal capillary bed in pregnancies complicated by IUGR with AED, which showed maldevelopment of fetoplacental capillaries (Krebs et al, 1996; Macara et al, 1996). All these data suggest that the defect might reside beyond the stem villi. On the other hand, relevant contribution of capillaries to overall vascular impedance is not in agreement with general views in cardiovascular physiology. Therefore, the debate continues (Trudinger, 1996) and is mainly due to unsatisfactory methodologics for visualising the fetoplacental vascular system.

Direct methods, such as three-dimensional reconstruction of the villous vessels from serial sections or from scanning electron micrographs (Kaufmann et al, 1985; Kaufmann et al, 1988, Leiser et al, 1985) of the villous branching patterns (Kosanke et al, 1993) are generally accurate. However, they are cumbersome and time consuming. Therefore they have always been applied to small probes which, due to placental heterogeneity (Mayhew and Burton, 1988) may not be representative. An alternative may be the use of a mathematical model based on topological analysis (Horsfield, 1978, 1990) applied to microvascular networks (Ley et al, 1986). Studies using this analysis on the placenta (Kosanke et al, 1993) show that the villous branching can be classified as a 'random segment branching' scheme. However, they have only been based on a few samples so far, because of the above mentioned reasons.

The most commonly used methods at present are indirect ones based on image analysis and quantitative evaluation of villous vessels and their calibre distribution in histological sections (Leiser et al, 1991; Macara et al, 1995; Jackson et al, 1995). However, the three-dimensional interpretation of such two-dimensional data is not straightforward and needs to be accurately tested.
Our study focuses on the following points.

1) Is there any difference in the distributions of the diameters of the stem villi arteries and arterioles when measured on histological sections from control and IUGR placentas?

2) Provided no difference is found (as in Macara study), does it mean that there are no differences in the stem villi vasculature, or that the diameter distribution is unable to show the difference satisfactorily?

This last hypothesis is examined by means of a multiparametric model, which is based partly on available morphometric data on vessel geometry and partly on assumptions. The effect of the parameters on the diameter distribution, and the relevant sensitivity, can be estimated by modifying the parameter values within the borderlines which are suggested by current placental stereological literature. The assessment of the relationship between the vessel diameter distribution predicted by the model and certain geometrical and topological properties of the villous tree vasculature (which are likely to be different in physiological and IUGR placentas) can shed much light on the usefulness of any comparative study based on the respective diameter distributions.

\section{MATERIALS AND METHODS}

We included 14 placentas in the study: eight from pregnancies complicated by preeclampsia (persistent blood pressure $\geq 140 / 90$ with proteinuria) and IUGR (birthweight below $10^{\text {th }}$ centile of the Italian reference curves (Parazzini et al, 1991) and six from age-matched normal preterm pregnancies.

In all cases an ultrasound scanning was performed within 48 hours of delivery. Fetal biparietal diameter, abdominal circumference and femur length were measured; amniotic fluid volume and fetal movements were assessed. Doppler flow velocity waveforms were recorded from the uterine arteries and from the umbilical arteries of a free-floating loop of the umbilical cord. IUGR was defined as an abdominal circumference measurement below $10^{\text {th }}$ centile of 
our reference ranges (Nicolini et al, 1986). A value of the Resistance Index (RI) on the uterine arteries above 0.58 and of the Pulsatility Index (PI) on the umbilical artery above the 90 th centile of our reference ranges (Todros et al, 1996) were considered abnormal.

The eight placentas meeting these criteria were divided into two subgroups on the basis of the umbilical Doppler findings: in 4 cases the value was above the 90th centile with presence of end diastolic velocities (PED); in the other 4 cases there was absence of end diastolic velocities (AED). The $\mathrm{RI}$ on the uterine arteries was above the cut-off value in all cases.

The six normal placentas were derived from women who delivered preterm after an otherwise uneventful pregnancy; fetal growth had been normal and birthweight was always above 10th centile (Parazzini et al, 1991); the umbilical and uterine Doppler measurements were normal. The membranes had never been ruptured for longer than 12 hours before delivery and signs of amniotitis were also excluded by histological studies. All measurements were performed with an Aloka SSD 680 color Doppler equipment, with a $3.5 \mathrm{MHz}$ probe and the high pass filter set at 100 Hertz.

From each freshly delivered placenta three probes reaching from the chorionic to the basal plate were excised from three different parts of the placenta and fixed in phosphate buffered $4 \%$ formaldehyde solution for 12-24 hours. After fixation, the probes were dehydrated in graded series of ethanol and embedded in paraffin. Paraffin sections were stained with hematoxilin and eosin. Immunohistochemical reaction with a monoclonal antibody binding to alfaactin was used to stain the arteries of the stem villi. The arteries were then counted and their mean diameter was measured by means of a morphometer connected to the light microscope (Zeiss) through a high resolution videocamera (JVC). The distribution of the diameters and the number of arteries per $100 \mathrm{~mm}^{2}$ of villous tissue were then determined for each placenta. A "branching index" was derived as follows. Usually there is only one artery per villus, but sometimes it is possible to see two or more arteries. It is maintained that in these cases the artery has already branched (and the villus is going to branch just below). The branching index was calculated as the proportion of stem villi with two or more arteries (Todros et al, in press).

In order to compare groups, the Kruskal-Wallis test and the Mann-Whitney test with Bonferroni correction for multiple comparisons were used. Diameter distributions were compared using the $\chi^{2}$ test. Differences were considered significant at $p<0.05$.

\section{MATHEMATICAL MODELING}

\section{Basic Assumptions}

The topological aspects of an organ can be described by a mathematical model, provided it is realistic enough to allow the comparison between the main characteristics of the organ and the corresponding model hypotheses.

The villous arterial system is a dichotomously branching structure embedded in the stromal tissue of placental villi. It exceeds from the largest stem villi (trunci chorii) to the smallest stem villi (rami and ramuli chorii) and is fed by arteries and arterioles. Further smaller branches, represented by intermediate, mesenchymal and terminal villi (Figure 1), are to be found in the most peripheral parts of the villous tree, and are fed by capillaries. Accordingly, the theoretical arterial tree can be described by the total number of generations of vessels $N_{g e n}$ starting from the largest (generation index $i=1$, corresponding to the trunchus chorii artery, of cross sectional area $A_{0}$ ) to the smallest stem villi arterioles (generation index $i=N_{\text {gen }}$ ). In our model villous arteries and arterioles are assumed to have the same length, irrespective of the generation to which they belong, whilst diameters are assumed to depend on the generation. We allowed a certain distribution of the values to avoid a description which is too schematic (with all the vessels of the same generation having exactly the same diameter). We assumed 


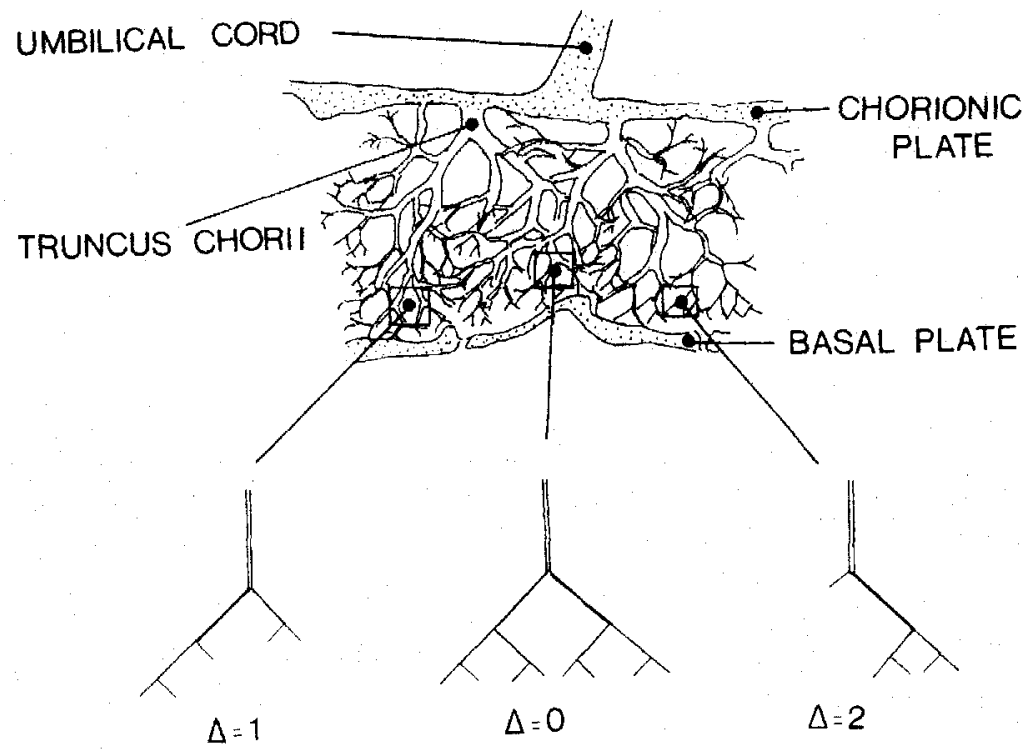

FIGURE 1 A schematic drawing of the placental structure. Magnified view of the terminal part of a villous tree with asymmetry $\Delta=1,0$ and 2 respectively at the first bifurcation is shown.

a Gaussian distribution centered at the 'theoretical' value of the diameter with a relative dispersion $\Sigma_{r}$.

A slightly asymmetric branching pattern was assumed for the most peripheral branching generations (Piantà et al, 1995). This is in agreement with observations obtained from vessel casts (Leiser et al, 1985). The model compares situations in which the asymmetry starts at different generations since a considerable degree of asymmetry has been observed especially for the central positioned branching generations (Demir et al., 1997). The generation at which asymmetry is assumed to begin is given by the parameter $N_{d e l}$; asymmetry itself is described by the parameter $\Delta$, whose value is changed randomly within a given range at each branching point.

In order to estimate the diameter distribution expected on an intersecting plane, we assumed that the villous tree can be inscribed into a hemisphere of radius $R$, where the concentric shells of constant thickness $r$ are progressively filled by vessels of successive generations (Figure 2). The distance $h$ between the origin of the tree (corresponding to the point of insertion of the truncus chorii at the chorionic plate) and the sectional plane (perpendicular to the chorionic plate) had to be introduced as a new parameter in the model. In our model we used the ratio $h / R$, which defines the relative distance at which the tree is intersected precisely $(h / R=0$ describes intersection at the truncus chorii level, $h / R=1$ describes an intersection perpendicular to the most peripheral stem villi and their vessels).

A more detailed description is given in the Appendix.

\section{Cross Validation of the Model}

Ten perfusion-fixed term human placentas derived from clinically normal pregnancies were used to validate the model. Histological sections were obtained with the methods previously described.

The distribution of the vessel calibers of each slide was compared with 19305 different modelpredicted distributions by changing the parameters values as follows: $h / R$ varies from 0.25 to 0.85 with steps of $0.05, \Sigma_{r}$ varies from 0.15 to 0.25 with steps 


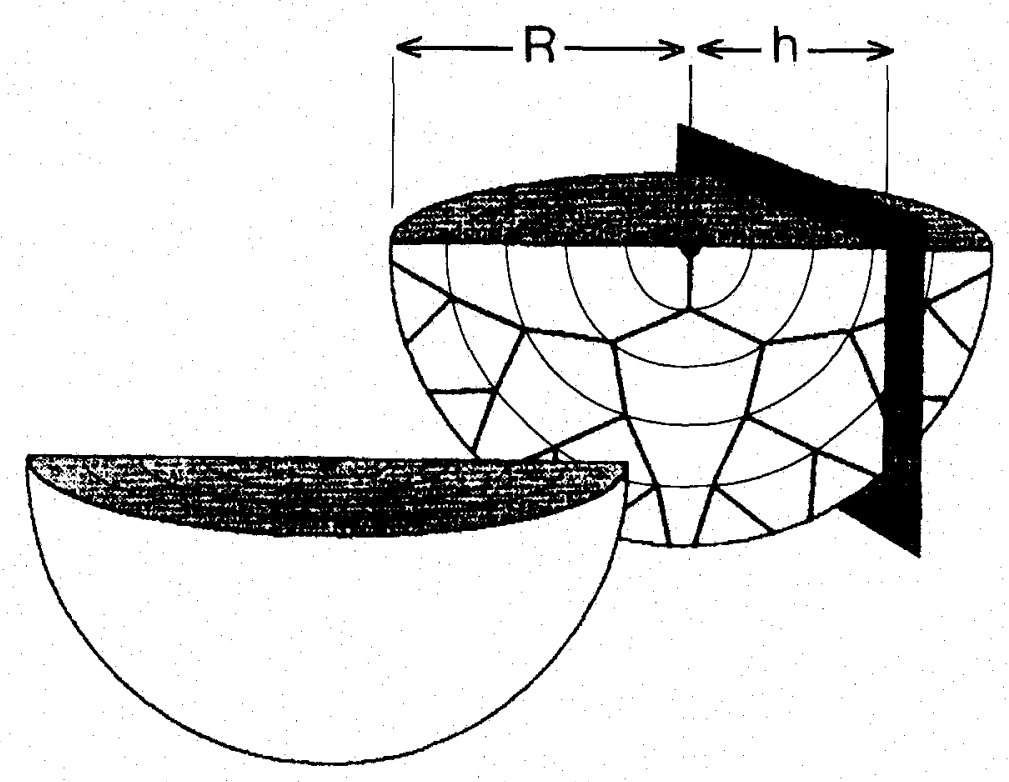

FIGURE 2 Model of the villous tree showing vessels branching as related to the concentric shells of a placental lobule, which has been simplified as an hemisphere. The point shaded surface corresponds to the chorionic plate and the intersecting plane to the slide on which morphometric measurements are performed. $h=$ distance between the origin of the tree and the sectional plane; $R=$ radius of the hemisphere or villous tree.

of $0.01 ; A_{0}$ varies from 0.003 to 0.007 with steps of $0.001 ; N_{\text {gen }}$ varies from 13 to 15 with steps of $1, \Delta$ varies randomly between 0 and 2 and finally $N_{\text {del }}$ varies from 7 to 15 with steps of 1 .

The best fit was assessed by the $\chi^{2}$ test: the estimated parameter values are accepted when the histogram of the deviations between the experimental and the 'predicted' diameter distribution is gaussian (null hypothesis) at the $5 \%$ confidence level.

The influence of each parameter on the distribution of the vessel calibers was assessed by changing each parameter value individually while keeping the other parameters fixed at their standard value (chosen according to current placentologic knowledge); this is called sensitivity analysis. A parameter is considered to be sensitive if a one-step change produces a statistically significant change in the calculated diameter distribution, as assessed by its mean value. The more steps that are needed to induce a statistically significant change, the less sensitive is the parameter.

\section{RESULTS}

\section{Normal and IUGR Placentas}

The three groups were comparable for gestational age at birth. Placental weight was lower in PED and AED groups, although placental weight/birthweight ratio was not significantly different (Table I).

The arterial count was lower in the AED group (Table II). The reduction affects all the generations of stem villi, from the largest to the smallest ones. Indeed, the distribution of the vessel

TABLE I Fetal and placental characteristics (mean \pm SD)

\begin{tabular}{lcccc}
\hline & GA & BW & PW & PW/BW \\
\hline Control & $31 \pm 0.3$ & $1850 \pm 620$ & $480 \pm 56$ & $0.30 \pm 0.15$ \\
PED & $34 \pm 1.5$ & $1395 \pm 120$ & $270 \pm 32^{*}$ & $0.19 \pm 0.02$ \\
AED & $31 \pm 3.7$ & $1060 \pm 470$ & $245 \pm 31^{*}$ & $0.25 \pm 0.09$
\end{tabular}

PED: present end-diastolic flow; AED: absent end diastolic flow; $\mathrm{GA}=$ gestational age, $\mathrm{BW}=$ Bitth weight, $\mathrm{PW}=$ Placental weight. * significant $(p<0.05)$ compared to controls 
TABLE II Artcrial count (mean \pm SD)

\begin{tabular}{lll}
\hline & Art/100 $\mathrm{mm}^{2}$ & Branching Index \\
\hline control & $352 \pm 60$ & $0.19 \pm 0.05$ \\
PED & $330 \pm 130$ & $0.16 \pm 0.05$ \\
AED & $270 \pm 38$ & $0.09 \pm 0.03^{*}$
\end{tabular}

PED: present end-diastolic fow; AED: absent end diastolic flow:

"significan compared to controls

diameters was not different in the three groups. The branching index was significantly lower in the AED group (Table II). There was no difference in the diameter distributions either between control and IUGR groups or between the two IUGR groups.

\section{Mathematical Model}

Diameter distributions theoretically predicted by the model were compared with the diameter distribution obtained from sections of 10 normal placentas. Figure 3 shows an example, with the black lines representing the experimental data and the empty columns representing the theoretical data.

The parameter values and their sensitivity are reported in Table III.

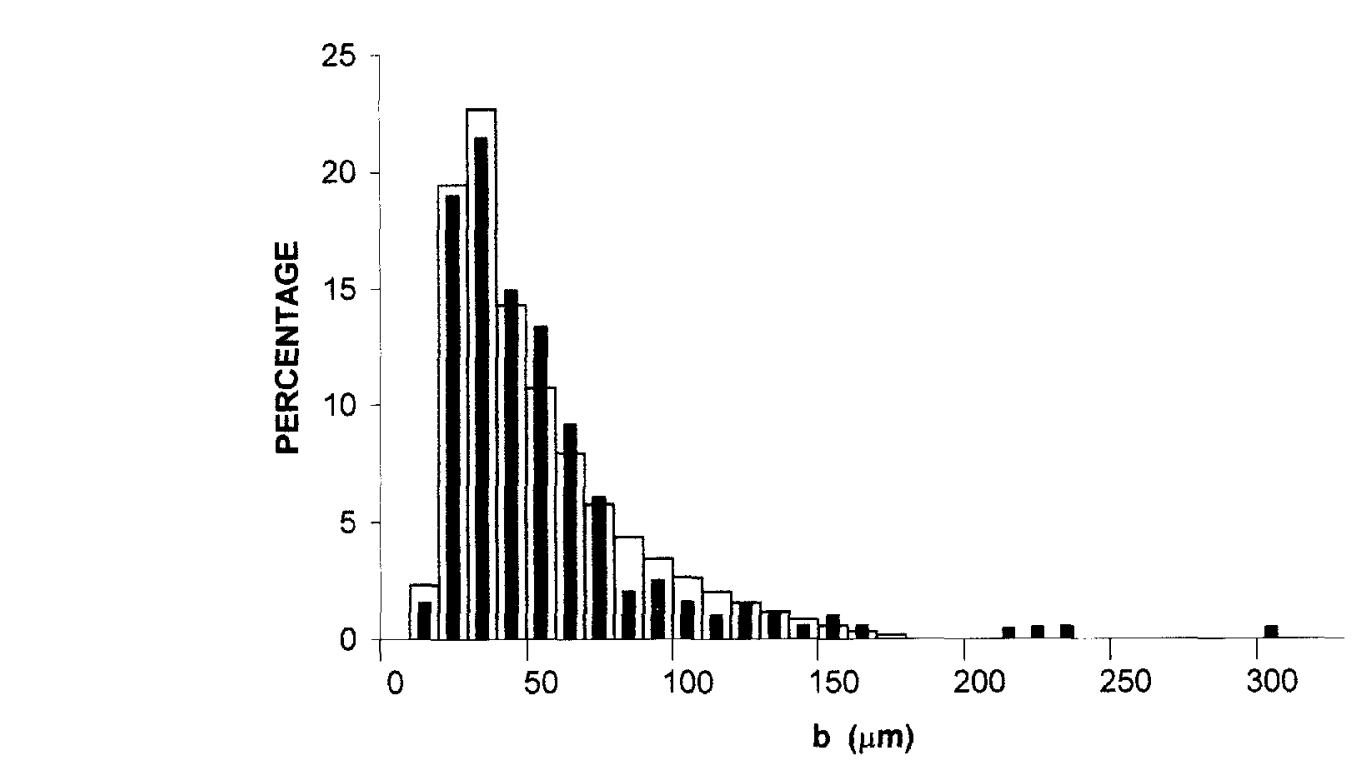

TABLE III Estimation of parameters and their sensitivity based on normal placentas

\begin{tabular}{lc}
\hline Parameter & Mean $\pm \mathrm{SD}$ \\
\hline$h / R^{\circ}$ & $0.53 \pm 0.09^{*}$ \\
$\Sigma_{r}$ & $0.24 \pm 0.02^{*}$ \\
$\Lambda_{0} \mathrm{I} 0^{-3} \mathrm{~m}^{200}$ & $3.5 \pm 0.7$ \\
$N_{\text {set }}{ }^{\circ 0}$ & $14.1 \pm 0.9$ \\
$N_{\text {det }}$ & $11.2 \pm 2.5^{*}$ \\
\hline
\end{tabular}

*indicates significant $(p<0.05)$ difference of the parameter value among the 10 slides:

slightly sensitive parameter (the diameter distribution is significantly $(p<0.05)$ affected only by at least two stepwise changes in the parameter value);

${ }^{\infty}$ sensitive parameter (the diameter distribution is significantly ( $p$. 0.05 ) affected by a stepwise change in the parameter value)

TABLE IV Results gainod when applying the model to normal and IUGR placentas (mean \pm S.D.)

\begin{tabular}{lccc}
\hline Parameter & Control & PED & AED \\
\hline$h / R$ & $0.53 \pm 0.13$ & $0.56 \pm 0.06$ & $0.52 \pm 0.10$ \\
$\sum_{r}$ & $0.18 \pm 0.01$ & $0.21 \pm 0.03^{*}$ & $0.22 \pm 0.02^{*}$ \\
$A_{0} 10^{-3} \mathrm{~m}^{2}$ & $3.2 \pm 0.5$ & $3.00 \pm 0.01$ & $3.5 \pm 1.0$ \\
$N_{g e n}$ & $14.4 \pm 0.9$ & $14.5 \pm 0.6$ & $14.3 \pm 0.5$ \\
$N_{d i l}$ & $8.6 \pm 3.6$ & $9.0 \pm 2.2$ & $9.8 \pm 3.4$ \\
\hline
\end{tabular}

PED: present end-diastolic flow: AED: absent end diastolic flow: *indicates significant $(p<0.05)$ difference of the parameter value.

FIGURE 3 Comparison between the morphometric diameler distribution (filled bars) from a slide (human term perfused placenta) and the model-predicted one (empty bars). The parameter values obtained with the best fit procedure are: $h / R=0.4, \Sigma_{r}=0.20$. $A_{0}=0.004 \mathrm{~cm}^{2}, N_{d d}=13, N_{g e n}=15$. random asymmetry and $\chi^{2}=4.56$. (see text for explanation). 
The results of applying the model to the control and IUGR placentas are reported in Table IV.

\section{DISCUSSION}

We found no difference in arterial count and in stem villi artery diameter distribution between control and IUGR placentas of the same gestational age. This result agrees with that of Macara et al (1995).

When applying the model to the normal placentas we found differences for 3 of the parameters $(h / R$, $\Sigma_{r}$ and $\left.N_{d e^{\prime} l}\right)$, while when applying the model to the control and IUGR placentas the only parameter significantly different was $\Sigma_{r}$. The sensitivity analysis shows that only two of the parameters $\left(N_{g e n}\right.$ and $A_{o}$ ), induce significant variations in the predicted diameter distribution. The diameter distribution is relatively insensitive to changes in $h / R . \Sigma_{r}$ and $N_{d e l}$ do not affect the distribution significantly.

We conclude that those parameters $\left(h / R, \Sigma_{r}, N_{\text {del }}\right)$ which yield physically significant differences in the distribution of the diameters of the villous arteries do not undergo a statistically significant change between normal and IUGR placentas.

The fact that only two of the model parameters show good sensitivity can reflect both:

1) an intrinsic inability of the model in describing the vascular system or, more likely,

2) the fact that a single experimental variable (the vessel diameter distribution) cannot give complete information about the $3 \mathrm{D}$ structure of the stem villi vasculature. We already suspected that vessel diameter distribution alone might be inadequate since no significant differences had been found by Macara et al (1995) when comparing physiological and pathological placentas.

As far as the first point is concerned, the main model inadequacies are the following:

- recent data (Demir et al, 1997), based only on a few reconstructed villi, suggest that a larger number of generations should be considered. However, if this were the case, the number of generations calculated by the model should always have been the maximum permitted, i.e. 16; we actually found a number of generations varying between 13 and 16 , according to older data.

- Again, recent data cast some doubts on the correctness of the two assumptions, i.e. the asymmetry is mild and it interest the more peripheral generations. It was observed that asymmetry is more severe and it appears in the very first generations (Demir et al, 1997). Our results do not provide information about the first issue. As far as the second point is concerned, they would agree with previous data. In fact, if asymmetry were already present in the first generations, the values of $N_{\text {del }}$ would pool around the minimal admitted values $N_{d e l}=7$, instead of varying between 7 and 12 .

- Finally, the assumption that all vessels have the same length is very unrealistic. We expect the actual villous tree to be asymmetric and the branches to have different lengths depending on the generation to which they belong. Differences in vessel length is also suggested by the computation of the 'branching index', showing a decreasing distance between successive branchings at increasing generations.

Unfortunately, when both asymmetry and varying vessel length are accounted for at the same time, the mathematical treatment becomes very difficult and unmanageable in analytical terms. It might be worth trying to solve the mathematical problem by numerical techniques, providing that the raw approximation we considered in this paper gives sensible results and the model proves to be useful.

With regard to the second point (that a single variable cannot capture the 3D structure of the stem villous vasculature) the fact that there is no significant difference in the diameter distributions between normal and IUGR placentas does not necessarily mean that there is no difference between the stem villi arteries vasculature. It may be that the vasculature is actually different but that the variable we have chosen (diameter distribution) is inadequate to show the differences. Recent studies on a few reconstructed villous vessel trees (Kosanke et al, 1993) suggest that it is the asymmetry itself that masks 


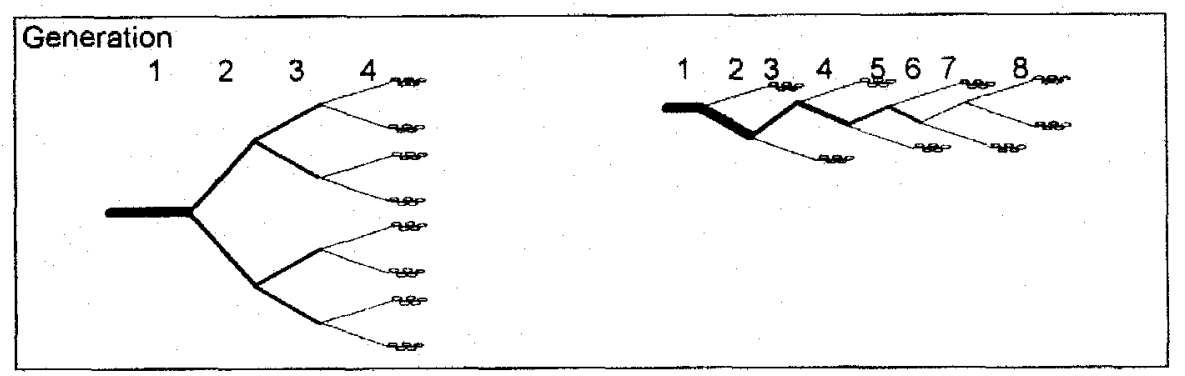

FIGURE 4 Two different vascular trees showing the same number of terminal villi and similar vessel diameter distributions.

the changes caused by IUGR. The two vascular networks depicted in Figure 4 show the same number of terminal villi (which are the preponderant part in the diameter distribution) and no great differences between two subsequent generations of stem villi in the asymmetric scheme. As a consequence, no considerable influence concerning the distribution of the vessel diameters can be detected by evaluating histological sections. It is possible that new parameters, such as the 'branching index', will prove more useful than the diameter distribution in differentiating the vasculature of normal and IUGR placentas.

\section{CONCLUSIONS}

The main conclusion proposed by the present study, based on both an experimental and a modelling investigation of the stem villi placental vascularization, is that arterial count and diameter distribution do not provide a useful way to distinguish between normal and IUGR placentas.

One possible explanation is that most of the qualitative and quantitative differences among normal and abnormal placentas are found at the level of the peripheral villi, which account for almost $2 / 3$ of the villous tissue (Todros et al, in press). The stem villous vasculature may or may not be affected in case of IUGR. Another (not alternative) explanation may be that there are too few larger vessels to detect a difference in arterial count or diameter distribution.

On the other hand the model shows that differences in symmetry and in geometry of the stem villous vessels do have little, if any, effect on the diameter distributions. As a consequence, any validation of the predictions of multiparametric models based on 'numerical fitting' is questionable as long as the diameter distributions are the only available data.

Since the information from $2 \mathrm{D}$ histological sections obtained and processed up to now, i.e. vessel number and section, does not differentiate the vascular structures, should we conclude that only direct $3 \mathrm{D}$ reconstruction of the villous tree is reliable? In principle, new parameters related to some 'intrinsic' properties of the villous tree vasculature, such as the branching characteristics, could be examined on 2D histological sections. Our experimental results show that the 'branching index', for instance, is different in AED and PED placentas. Further investigation is needed, however, based both on measuring and modeling the placental vasculature, to better clarify the problem.

\section{APPENDIX}

Dichotomy implies that any parent vessel (generation index $i$ ) generates two daughter vessels (generation index $i+1$ ).

\section{Cross sectional area}

The cross sectional areas $a_{i}$ of parent and $a_{i+1}$ of daughter vessels:

$$
a_{i+1}=\frac{K}{2} \cdot a_{i}
$$


with $K$ being the constant ratio between total crosssectional areas of dichotomously branching vessels belonging to two subsequent generations. Based on the data previously published (Leiser et al, 1991), $K$ is assumed to be 1.3 (Piantà et al, 1995). Vessels belonging to different generations are assumed to have the same length (Leiser et al, 1991).

\section{Vessel asymmetry}

Branching is asymmetric if the daughter vessels have different cross sectional areas. The degree of asymmetry can be defined for each branching point by the asymmetry parameter $\Delta$ (Piantà et al, 1995) (Figure 1). $\Delta$ is the difference between the generation index of two daughter vessels generated by the same parent vessel. It may have the same value at each bifurcation (tree asymmetry equal to $0,1,2, \ldots$ ) or it may change randomly (random tree asymmetry). This latter possibility seems to be the more realistic one, and therefore is the one applied in this study. $\Delta$ is randomly chosen between 0 and 2 at each branching point.

\section{Sectioning procedure}

To model the sectioning procedure in the simplest way, we assumed that a symmetric vascular tree can be inscribed in a hemisphere formed by $N_{\text {gen }}$ concentric shells of thickness $r$. The vessels of the last generation (the most peripheral ones) fill the external shell of the hemisphere. We assume furthermore that all the vessels belonging to a given generation are uniformly distributed in their spherical shell and intersect the shell perpendicularly. The assumptions that all the vessels belonging to a given generation have the same length and are disposed radially (so that their length $l$ is equal to the hemispheric shell thickness $r$ ) seem to be quite unrealistic, but are necessary in order to keep the mathematics to a manageable level.

When a plane (Figure 2) intersects the hemisphere perpendicularly to the 'chorionic plate' at a distance $h$ from the center, it crosses a number of vessels belonging to different shells of the tree. A rough estimate of their number can be obtained if the oversimplifying assumption that all the vessels are radially disposed is made. The total number of vessels cut from a plane is then given by the sum of the vessels intersected by the plane in each shell, and namely:

$$
\sum_{k=k_{\min }}^{N_{\text {teth }}} n(k)=\sum_{k=k_{\min }}^{N_{\text {gin }}} \rho(k) \cdot v(k)
$$

where $\rho(k)$ is the vessel density:

$$
\rho(k)=\frac{N(k)}{V(k)}
$$

being $N(k)$ the number of vessels belonging to the $k$-th generation, $V(k)$ the volume of the $k$-th shell, and $v(k)$ the volume of the portion of the $k$-th shell intersected by the plane defined by the spheres of the $k$-th and $(k-1)$-th orders (of radii $r(k)$ and $r(k-1)$ ) and the solid angle under which the origin sees the intersecting plane.

When the first intersected shell is considered ( $k=k_{\min }$, where $k_{\min }$, is the first integer number following $h / r(k)$ ), the above portion is a spherical segment, while for the other shells it is the volume obtained by subtracting the two spherical sectors whose radii are $r(k)$ and $r(k-1)$.

The number of vessels intersected by the slide in the $k$-th shell is therefore given by

$$
n(k)=\frac{N(k) R}{2} \cdot \frac{h / R}{r(k) r(k-1)}
$$

or, for the first shell intersected by the plane,

$$
n\left(k_{\min }\right)=\frac{N\left(k_{\min }\right)}{2} \cdot\left[1-\frac{h / R}{R r\left(k_{\min }\right)}\right]
$$

The leading parameter is the quantity $h / R$ expressing the distance of the intersecting plane from the center of the hemisphere referred to its maximum radius $R$. In the asymmetric case vessels of the same generation may belong to different orders, and, provided they have the same length, corresponding to the given shell thickness, the above procedure must be separately applicd to each order represented in the given generation.

When the number of 'intersected' vessels of each generation is evaluated, the model is able to predict 
the diameter histograms by associating the corresponding vessel diameter computed by equation (1) with the above frequencies.

\section{GLOSSARY}

$A_{0}=$ diameter of the artery of the truncus chorii

$N_{g e n}=$ total number of branching generations

$a_{i} / a_{i+1}=$ ratio:diameter of parent/diameter of daughter artery

$K=$ ratio of sectional area of two arteries belonging to two generations following each other (here: 1.3)

$N_{d e l}=$ generation at which the random asymmetry starts

$\Sigma_{r}=$ (standard deviation/mean) of the gaussian distribution of diameters of arteries belonging to the same branching generation $\Delta=$ asymmetry parameter

$h=$ distance of the plane of section from the insertion of the truncus chorii

$R=$ radius of the external ideal hemisphere surrounding the villous tree

$h / R=$ ratio between $h$ and $R$

$r=$ thickness of the shells of the hemisphere

$l=$ length of the vessel segments crossing the shell $(=r)$

$\rho(k)=$ density of vessels in the $k$-th shell of the hemisphere

$N(k)=$ total number of vessels belonging to the $k$-th generation

$V(k)=$ volume of the $k$-th shell of the hemisphere being cut

$v(k)=$ portion of the $k$-th shell intersected by a defined plane

$r(k)=$ external radius of the $k$-th shell of the hemisphere

\section{Acknowledgements}

This work has been supported by the European BIOMED Project, (Concerted Action C.BMHACT98-3621) the Italian Research Council (CNR) (Bilateral project, n.93.04039.CTO4) and by the German Research Council (DFG) (Ka 360/7-1,2,3).
We thank Prof. P. Rossi and Dr. A. Cadario from Dip. Scienze Cliniche e Biologiche of the University of Torino ( $\mathrm{S}$. Luigi) for making available the equipment for morphometric measurements.

\section{References}

Demir, R., Kosanke, G., Kohnen, G., Kertschanska, S. and Kaulmann, P. (1997) Classification of human placental stem villi: Review of structural and functional aspects. Microsc. Res. Techn., 38, 29-41.

Giles, W. B., Trudinger, B. J. and Baird, P. J. (1985) Fetal umbilical artery fow velocity waveforms and placental resistance: pathological correlation. Br. J. Obstet. Gyncecol., 92, 31-38.

Horsfield, K. (1978) Morphometry of the small pulmunary arteries in man. Circ. Res., 42, 593-597.

Horsfield, K. (1990) Diameters, generations and orders of branches in the bronchial tree. J. Appl. Physiol, 68, 457-461.

Jackson, M. R., Walsh, A. J., Morrow. R. J., Mullen. J. B. M.. Lye, S. J. and Ritchie, J. W. (1995) Reduced placental villous trec claboration in small-for-gestational-age pregnancies: relationship with umbilical artery Doppler waveforms. Am. J. Obstet. Gynatol., 172, 518-525.

Kaufmann, P., Bruns, U., Leiser, R., Luckhardt, M. and Winterhager, E. (1985) The felal vascularization of term human placental villi. H. Intermediate and tominal villi. Anat. Embrol. (Berl.). 173, 203-214.

Kaufmann, P., Luckhardi. M. and Leiser, R. (1988) Threedimensional representation of the letal vessel system in the human placenta. Trophoblast Res., 3, 113-137.

Kingdom, J. C. P., Rodeck, C. and Kaufmann. P. (1997) Commentary: umbilical artery Doppler-more harm than good? $\mathrm{Br}$. J. Obstet. Gynaecol, 104, 393-396.

Kosanke, G., Castellucci, M., Kaufmann, P. and Mironov, V. A. (1993) Branching patterns of human placental villous trees: perspectives of topological analysis. Placenta, 14, 591-604.

Krebs, C., Macara, L. M., Kingdon, J. C. P., Bowman, A. W.. Greer, I. A and Leiser, R. (1996) Intrauterine growth restriction with absent end-diastolic flow velocity in the umbilical artery is associated with maldevelopment of placental cominal villous tree. Am. J. Obstet. Gynaecol., 175, 1534-1542.

Leiser, R., Luckhardt, M., Kaufmann, P., Winterhager, E. and Bruns, U. (1985) The fetal vascularisation of term human placental villi. I. Peripheral stem villi. Anat. Enbryol., 173. $71-80$.

Leiser, R., Kosanke, G. and Kaufmann. P. (1991) Human placental vascularization. Structural and quantitative aspects. 'Placenta: Basic Research for clinical application'. Soma $\mathrm{H}$ (ed), Karger, Basel.

Ley, K., Pries, A. R. and Gaehtgens, P. (1986) Topological structure of rat mesenteric microvessel network. Microv. Res., 32 $315-332$.

Macara, L., Kingdom, J. C. P., Kohnen, G., Bowman, A. W., Greer, I. A. and Kaufmann, P. (1995). Elaboration of stem villous vesscls in growth restricted pregnancies with abnomal umbilical artery Doppler waveforms. Br. J. Obstet. Gynaecol., 102, $807-812$

Macara, L., Kingdom, J. C. P., Kalumann, P., Kohnen, G., Hair, J., More, I. A. R., Lyall, F. and Greer, 1. A. (1996) Structural analysis of placental terminal villi from growth-restricted pregnancies with abnormal umbilical artery Doppler waveforms. Placenta, 17, 37-48. 
Mayhew, T. M. and Burton, G. J. (1988) Methodological problems in placental morphometry: apologia for the use of stereology based on sound sampling practice. Placenta, 9, $546-581$.

Nicolini, U., Todros, T., Ferrazzi, E., et al. (1986) Curve trasversali dell accrescimento fetale. Studio multicentrico. Minerva Gin: 38. 873-85.

Parazzini, F., Cortinovis, I., Bortolus, R. and Fedele, L. (1991) Standard di peso alla nascita in Italia. Ann. Ost. Gin. Med. Perin.: CXII, $203-246$.

Piantà, P. G.. Guiot. C. and Todros. T. (1995) Sudy of a regional circulatory system based on the "equivalent tube" concept: the feto-placental circulation. J. Biol. Res., 71, 61-67.

Todros, T., Ronco, G., Fianchino, O., et al. (1996) Accuracy of the umbilical arteries Doppler flow velocity waveforms in detecting adverse perinatal outcomes in a high risk population. Acta. Obstet, Gynecol. Scand., 75, 113-119.
Todros, T., Sciarrone, A., Piccoli, E., et al. Umbilical Doppler waveforms and placental villous angiogenesis in pregnancies complicated by fetal growth restriction. Obstet. Gynecol., [in press].

Trudinger. B. J., Giles, W. B., Cook. C. M.. Bombardieri, J. and Collins, L. (1985) Fetal umbilical artery flow velocity waveforms and placental resistance: clinical significance. $\mathrm{Br} . \mathrm{J}$. Obstet. Gynaecol, 92, 23-30.

Trudinger, B. J., Stevens, D., Connelly, A., et al.(1987) Flow velocity waveforms and the placental resistance: the effect of embolization of the umbilical circulation. Am. J. Obstet. Gynaecol., 157, 1443-1449.

Trudinger, B. J. (1996) Correspondence to: Macara ef al: Elaboration of stem villous vessels in growth restricted pregnancies with abnormal umbilical artery Doppler waveforms. Br. J. Obstet. Gynaecol., 103. 487-488. 


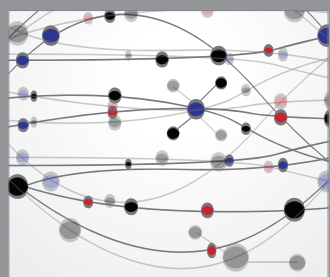

The Scientific World Journal
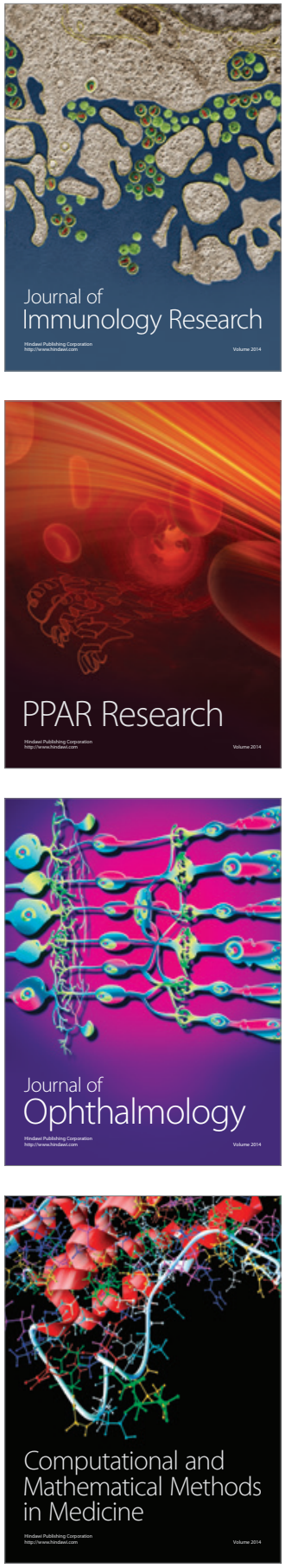

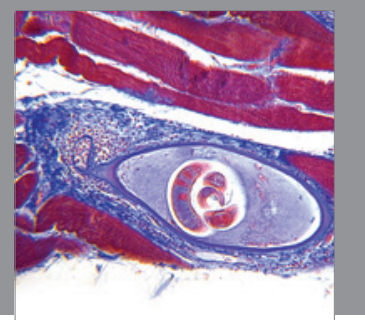

Gastroenterology

Research and Practice
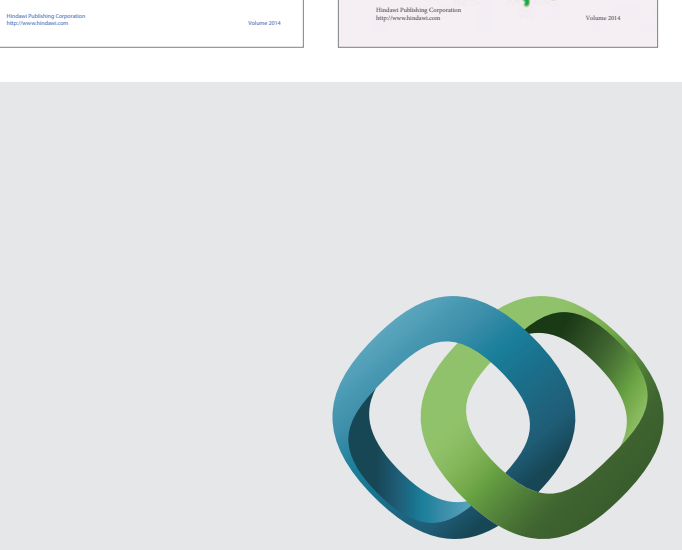

\section{Hindawi}

Submit your manuscripts at

http://www.hindawi.com
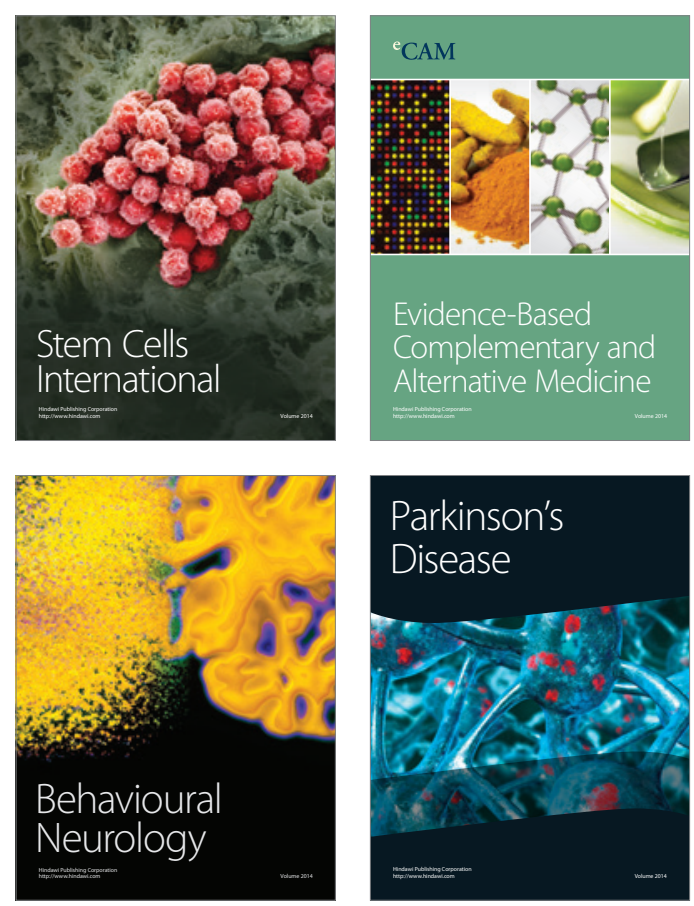

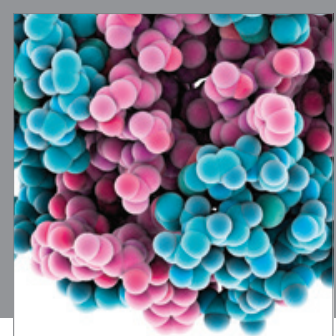

Journal of
Diabetes Research

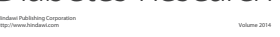

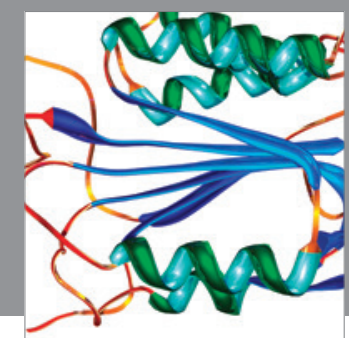

Disease Markers
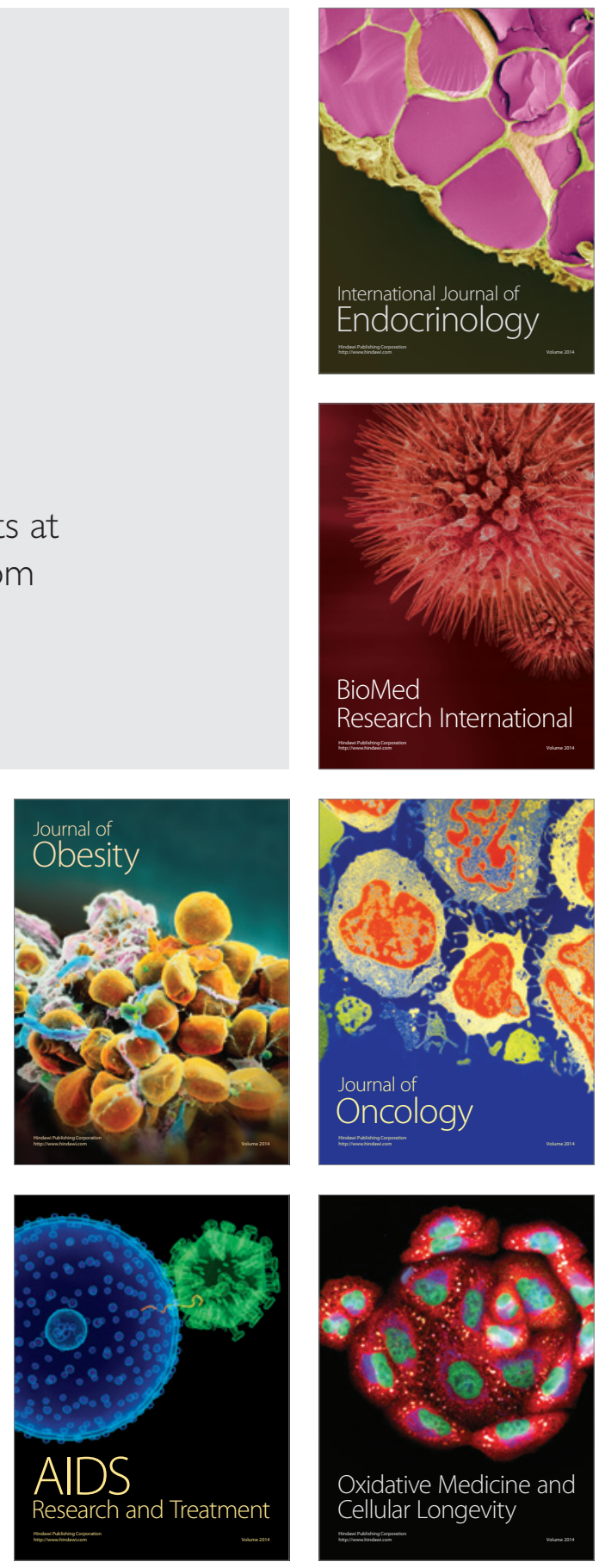